\title{
105. On the Ultra-violet Light Eclipse at the Upper Ionized Layer, in a Solar Eclipse of Sept. 21st, 1941.
}

\author{
By Takao Sato. \\ Mukaiyama Observatory, Tohoku Imperial University, Sendai. \\ (Comm. by A. Tanakadate M.I.A., Nov, 12, 1940.)
}

1. It is an extensively advocated doctrine that the earth-current and the earth's magnetism are caused by the induced current of ion at the two ionized layers in the atmosphere. With regard to these layers in the earth's atmosphere, Chapman has suggested that the upper one, at a height of about $220 \mathrm{~km}$., is ionized by ultra-violet light, while the lower, at about $100 \mathrm{~km}$. height, is ionized by neutral solar corpuscules.

If we assume this supposition to be true, when the ultra-violet light or corpuscules from the sun do not reach the ionized layers owing to their obscuration by the moon, as in the case of a solar eclipse, it would be naturally expected that the earth-current and the earth's magnetism will be also influenced on this occasion.

For this reason, geophysists attach great importance to a solar eclipse on account of its being an opportunity to verify the source of the earth-current and the earth's magnetism. At the time of the recent solar eclipse of June 19th, 1936, Ass. Prof. Y. Kato ${ }^{1)}$ issued an interesting investigation of this problem. In the present paper apart from discussing these physical problems the writer has applied the method of the computation of ordinary eclipses to the obscuration of the ultraviolet light.

2. Abbot and Milne have noticed that the ultra-violet light emitted from the solar disk presents a particularly conspicuous phenomenon of limb-darkening, diminishing its intensity steeply from about $85 \%$ of the radius of disk from its centre to the limb. Therefore, we can regard the obscuration of the above stated concentric circular disk as a total eclipse in this case.

To be brief, the obscuration of the above fictitious sun at the upper ionized layer in the earth's atmosphere, at a height of about $220 \mathrm{~km}$., is the characteristic of the ultra-violet light eclipse in a solar eclipse.

We consider an ellipsoid having $a_{1}=6598.388 \mathrm{~km}$. and $b_{1}=$ $6576.909 \mathrm{~km}$. as respectively its equatorial and polar radii, each of which is $22 \mathrm{~km}$. larger than that of earth. Then its flattening and eccentricity are given as follows.

$$
\frac{a_{1}-b_{1}}{a_{1}}=\frac{1}{307.2} \quad e=0.08068068
$$

The normal distance from this ellipsoid to the earth's surface is almost $220 \mathrm{~km}$. and not influenced by the latitude, so that this ellipsoid

1) Yosio Kato. Japanese Journal of Astronomy and Geophysies, Vol. XIV, No. 2, 1937. 
may be practically regarded as representing the surface of the upper ionized layer.

In the practice of computation, the coordinates of the centre of shadow and their variation and the coordinates of the point on the upper layer should be represented in units of the equatorial radius of the upper layer.

Again, the radius of the shadow on the fundamental plane must be, of course, represented in the converted unit.

3 . With respect to the projection on the earth's surface of the ultra-violet light eclipse of the upper ionized layer, the writer has ascertained that it is neglesible in the practical problem: that is to say, let the point on the upper ionized layer be $\left(x_{1} y_{1}\right)$ such that the inward normal at this point passes through $\left(x_{2} y_{2}\right)$ on the earth's surface then the geographycal latitudes of these two points are nearly equal.

4. The results of the computation of this eclipse are all exhibited in the map of Fig. 1. This eclipse has some different and interesting circumstances compared with the ordinary one of the same date.

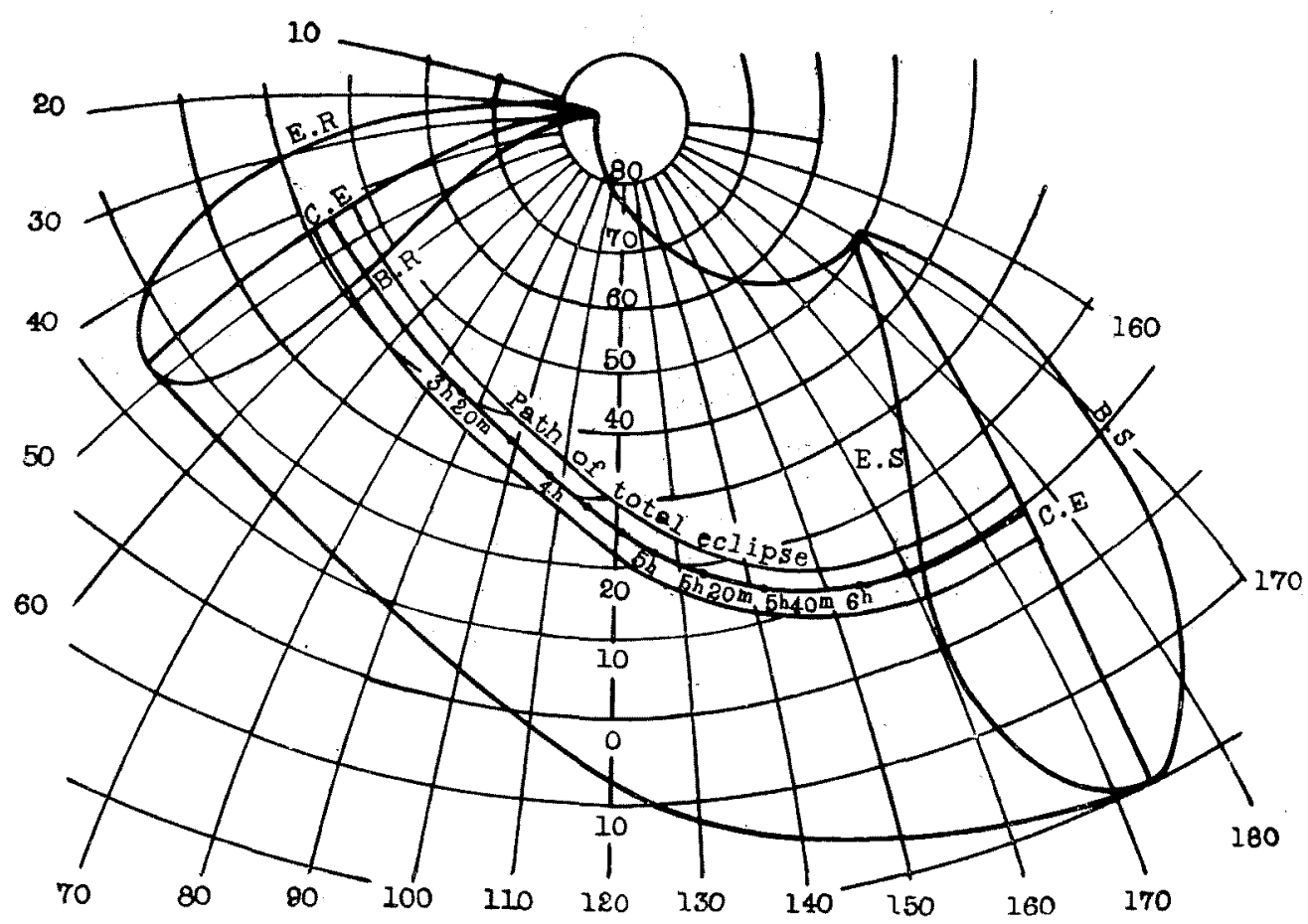

Fig. 1. Total ultra-violet light eclipse of 21st Sept. 1941.

E. R: Ends at sunrise

B. R: Begins at sunrise

E.S : Ends at sunset

B.S: Begins at sunset

C. E: Central eclipse

For reference, the map of the ordinary eclipse is shown in Fig. 2. In the ultra-violet eclipse, the rising and setting limits form two distinct curves, while in the ordinary eclipse they extend through the whole eclipse in north latitude, so that the former has both a northern and southern limiting curve, while the latter has only the southern 


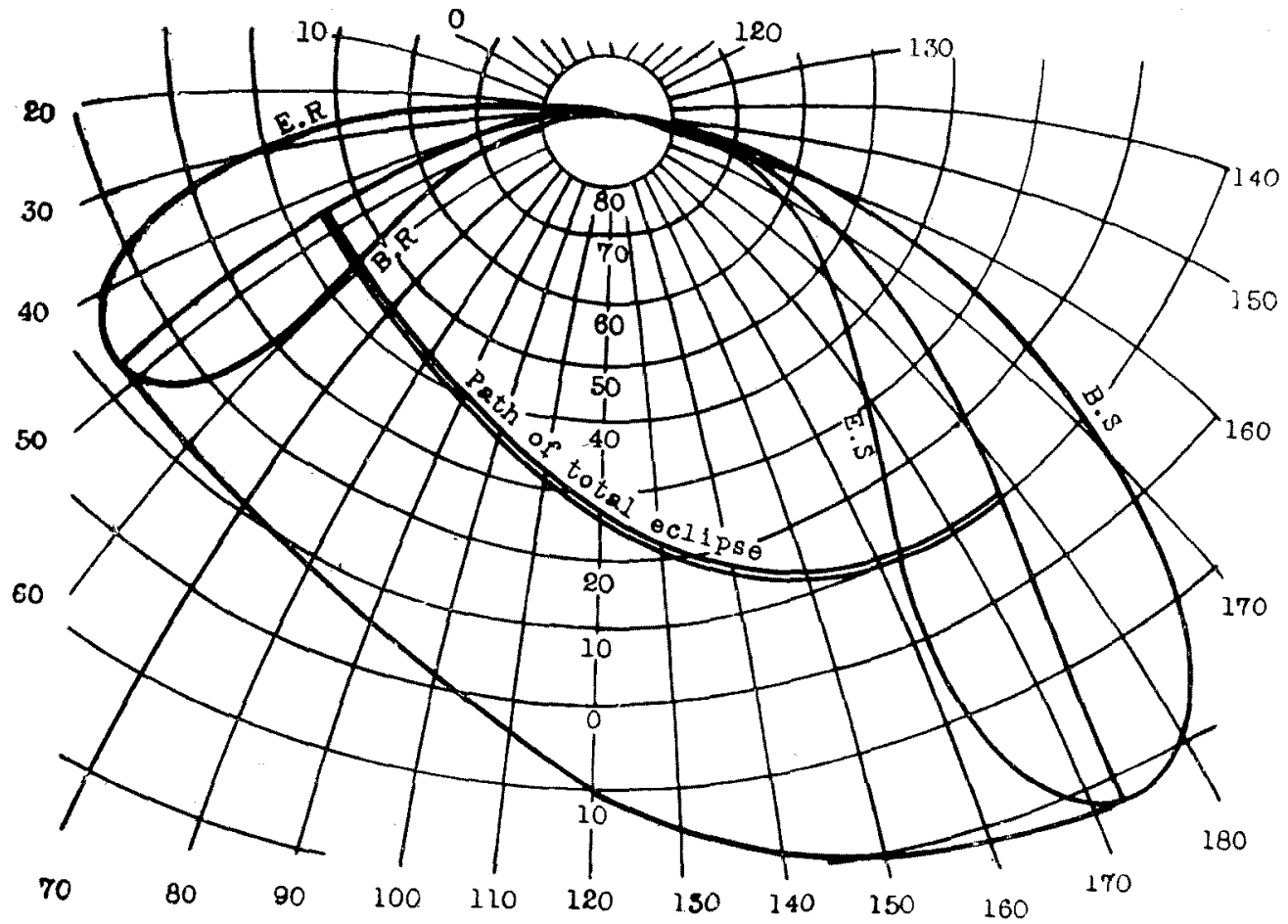

Fig. 2. Total eclipse of 21st Sept. 1941.

E. R : Ends at sunrise

B. R : Begins at sunrise

E. S: Ends at sunset

B.S: Begins at sunset

C. E: Central eclipse

limiting curve. These phenomena are indicating the following facts: in the ordinary sun (the true sun) the penumbral shadow passes over and beyond the north pole of the earth, while in the fictitious sunthe radius of this disk amounting to $85 \%$ of the true solar disk-the whole of the penumbral shadow is intersected with the upper ionized layer.

It is also characteristic of the ultra-violet eclipse that the outline of the closed curves of rising and setting limits is small compared with that of the ordinary eclipse. Next, as for the total zone which is most important for a practical observation, there is also a remarkable difference between the two eclipses, that is to say, first of all in the former the total zone is far more extensive than in the latter and moreover the former has a very long time of duration of totalityamounting to about 17 minutes at its maximum. Both these points of difference in the case of the ultra-violet eclipse may be, of course, attributed to the larger radius of umbra on the fundamental plane and accordingly also on the ionized layer. Again, the comparison of two places on the earth's surface, one place being that at which the latter central eclipse will occur at a given time and the other place being that at which the former one will occur on its upper ionized layer at the same time, is of great importance. Let $\vartheta$ be the hour angle of the vanishing point of the axis of the shadow at the given place. 
As the positive hour angle will be reckoned towards the west, and the negative towards the east, the eclipse will occur in the eastern heaven when $\vartheta<0$ and in the western when $\vartheta>0$. Accordingly at the same time the eclipse on the ionized layer will occur to the east or west of the eclipse on the earth according as $\vartheta<0$ or $\vartheta>0$. This inference has been numerically assured in our computation. Namely, the deviation of the place of the central eclipse of the ultra-violet light eclipse referring to that of the light eclipse of the same time is given as follows from the computed table of the latter given in the American Ephemeris.

$\begin{array}{cccccc}\text { G.C.T. } & 3^{\mathrm{h}} 20^{\mathrm{m}} & 3^{\mathrm{h}} 40^{\mathrm{m}} & 4^{\mathrm{h}} 0^{\mathrm{m}} & 4^{\mathrm{h}} 20^{\mathrm{m}} & 4^{\mathrm{h}} 40^{\mathrm{m}} \\ \Delta \omega & -3^{\circ} 05^{\prime} .8 & -1^{\circ} 24 .^{\prime} 8 & -0^{\circ} 31^{\prime} .6 & +0^{\circ} 03^{\prime} .3 & +031^{\prime} .7 \\ \Delta \varphi & -143.6 & -125.4 & -114.1 & -103.8 & -054.3 \\ \text { G.C.T. } & 5^{\mathrm{h}} 0^{\mathrm{m}} & 5^{\mathrm{h}} 20^{\mathrm{m}} & 5^{\mathrm{h}} 40^{\mathrm{m}} & 6^{\mathrm{h}} 0 \mathrm{~m} & \\ \Delta \omega & +0^{\circ} 59^{\prime} .3 & +1^{\circ} 31^{\prime} .8 & +2^{\circ} 22^{\prime} .0 & +4^{\circ} 48^{\prime} .9 & \\ \Delta \varphi & -045.4 & -037.0 & -028.5 & -018.9 & \end{array}$

As the longitude is taken as positive towards the west, and the latitude positive towards the north, the negative (positive) sign of $\Delta \omega$ shows the deviation towards the east (west), and the negative sign of $\Delta \varphi$ towards the south. Namely, in the northern hemisphere of the earth, owing to the situation of the sun in the southern heaven on this date, the deviation of $\varphi$ is of course towards the south.

This table, together with the sign of the computed value of $\vartheta$, will indicate a good coincidence with the above inference. Computing the values of $\Delta \omega$ and $\Delta \varphi$ at any time by interpolation from this table we shall be able to determine the places of the central eclipse on the ionized layer from The American Ephemeris.

5. In conclusion, the writer wishes to express his sincere thanks to Prof. T. Matukuma for his kind advices and to Prof. S. T. Nakamura and Ass. Prof. Y. Kato for their kind encouragements and criticisms in the course of this calculation. 